\title{
Energy optimization path planning for battery-powered agricultural rover
}

\author{
Cong Niu ${ }^{1}$, Xiutian Yan $^{1, a}$ \\ ${ }^{1}$ Department of Design Manufacture \& Engineering Management, University of Strathclyde, 75 Montrose Street, Glasgow G1 1XJ
}

\begin{abstract}
Battery life is critical for battery-powered agricultural rovers, so techniques such as optimized moving path planning are of great significance in this field. Finding an optimized path other than straight-line path could save energy and prolong the battery life. Compared with traditional straight-line path planning, an energy-optimized path planning is realized based on artificial potential field algorithm. In simulation studies, most of the uphill is avoided and at least $10.15 \%$ of energy is saved with the optimized path planning. We believe this energy optimization path planning algorithm is a feasible solution to extend the battery life for field operated agricultural rover.
\end{abstract}

\section{Introduction}

Battery-powered agricultural rover is a multifunctional robot platform that is often used in the field. This robot platform with suitable payload can do soil sampling, mapping, and fruit picking. But the energy of battery on board is very limited, and the battery life often determines the effectiveness and validity of the robot [1]. So how to extend the battery life of the robot has become an important topic in this field. Currently, there are hardly any work about path-planning for battery-powered agricultural rover from the perspective of energy optimization. With a more optimized path, the energy could be saved, and the battery life could be extended. To this end, an algorithm has been developed. The algorithm could be used on existing robot platforms which makes it cheaper than performing a hardware upgrade (Figure 1).

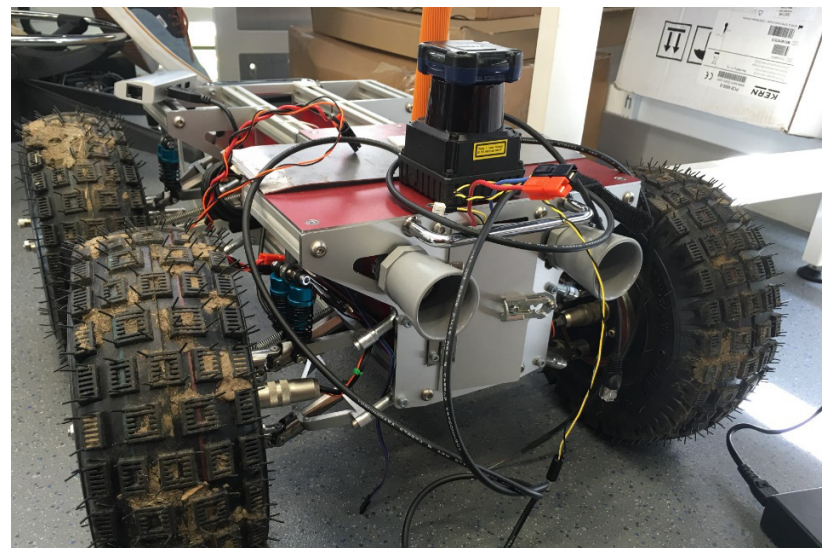

Figure 1. An agricultural rover

Normally the moving path of the rover is a straight line as it is the shortest distance. But sometimes a straight path is not the most energy efficient path when the terrain is not flat. Instead, going around the hills may be more energy efficient. By applying our path planning algorithm, a new path could be found. This generated path would save more energy than a straight-line path. When moving the rover at a fixed speed the energy spent on going up and down hill will be wasted as heat. Even with very high efficient energy recovery braking system, over 50 percent of the energy will be wasted. However, by going around the hill the energy could be saved.

\section{Conditions}

First presume when the rover climbs additional energy is needed when the kinetic energy cannot be converted back to the electric energy. The following variables can be set [2]. $E_{m}$ is total kinetic energy when moving. $E_{c}$ is the energy needed when changing the state of movement. $W_{r}$ is the work needed to overcome resistance. $P_{h}$ is the power of heat loss by the propulsion system. $P_{e}$ is the power needed for onboard electronic equipment. And $t$ is time.

The total energy required is

$$
E_{T}=E_{m}+E_{c}+W_{r}+t^{*}\left(P_{h}+P_{e}\right)
$$

The $E_{m}$ and $E_{c}$ are mainly dispensed on the mass of the rover. $P_{h}$ and $P_{e}$ are mainly dispensed on the motor, transmission and wheels. So for a given rover, what can be optimized is $W_{r} . W_{r}$ is composed of two parts, the work needed to overcome resistance when moving, and the work needed when in ascent stage. In descent stage $W_{r}$ could be counteracted by the gravity component in the direction of moving.

\section{Algorithm}

\footnotetext{
${ }^{\text {a }}$ Corresponding author: xiut.yan@gmail.com
} 
The algorithm is based on artificial potential field with modification and improvement [3]. Every environment variable has a corresponding artificial potential field calculated by an independent equation. Variables that can be calculated include topographic map and obstacle map. In our algorithm, two potential fields are considered, i.e., distance potential field and height difference potential field. The distance potential field is calculated based on terrain map. Suppose X and Y are horizontal and vertical coordinates for a given point. $\mathrm{X}_{d}$ and $\mathrm{Y}_{d}$ are horizontal and vertical coordinates for the destination point. $G$ is gravity, then the distance potential field Ep is calculated as follows.

$$
\mathrm{E} p=\mathrm{G}^{*} \sqrt{\left(\mathrm{X}-\mathrm{X}_{d}\right)^{2}+\left(\mathrm{Y}-\mathrm{Y}_{d}\right)^{2}}
$$

The height difference potential field is calculated based on topographic map and elastic potential energy formula. Suppose $\mathrm{H}_{P}$ is the height for a given point $\mathrm{p}, \mathrm{H}_{D}$ is the height of the destination point. Then the height difference potential field $\mathrm{E}_{p}$ is calculated as follows.

$$
\mathrm{E}_{p}=\frac{1}{2}\left|\mathrm{H}_{P}-\mathrm{H}_{D}\right|^{2}
$$

The two potential field can be combined by applying a potential field combining algorithm, i.e., weighted sum of the potential fields. Each potential field has an adjustment factor based on its importance. Based on the final combined potential field, a pathfinding algorithm is used to find the path for the rover by simulating a process of ball free scrolling from high potential energy point to lower point. An evaluation algorithm is utilized to assess how long is the path, how long is the path in ascent and is there any collisions with the obstacle [4].

The evaluation is performed iteratively. If any result of this evaluation has not reached all prerequisites, the algorithm will try different potential field modifiers which are defined based on the result of this evaluation and a new final potential field by the pathfinding algorithm. Then the process goes on until all requirements are achieved.

After the evaluation, another evaluation algorithm is utilized to calculate how much energy is saved with the optimized plan compared with the straight-line path. Then a series of equal difference potential field modifier will be tested, and the energy cost will be compared with each other, so an optimal path could be found [5].

\section{Simulation Energy}

By using the conditions previously mentioned, two simulation plans were generated with MATLAB software. Figure 2 are paths found by straight path planning and our energy optimization path planning respectively. The starting point is on the top left and the destination is on the bottom right.

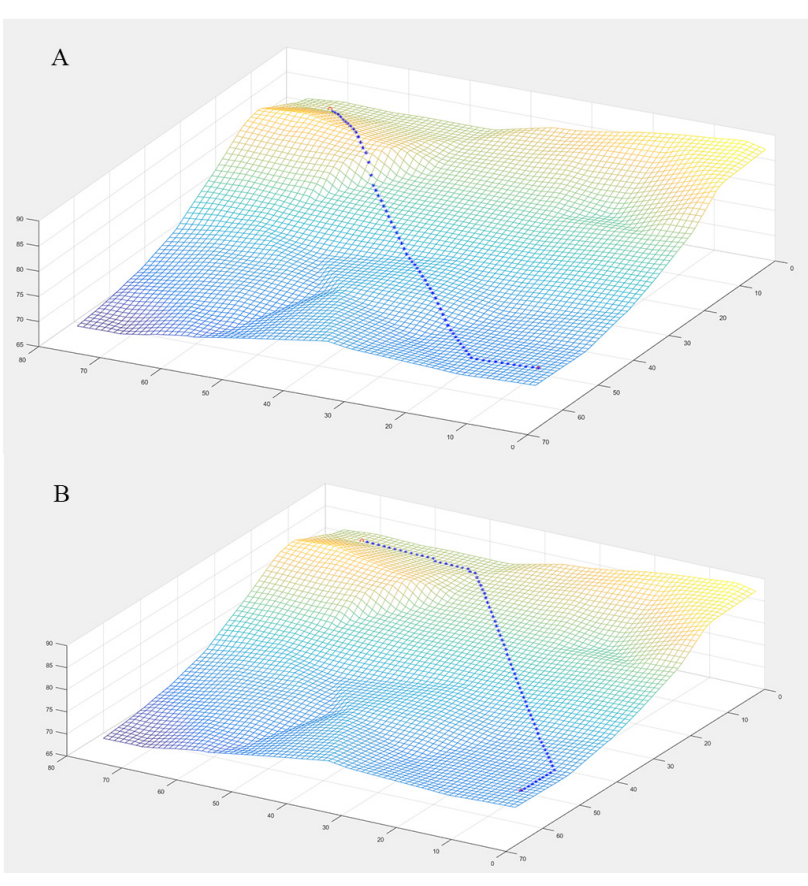

Figure 2. Path found by straight path planning (A) and our energy optimized path planning (B).

The diagram of the height-position relationship of these two paths is generated, as shown in Figure 3. Based on the diagram, the energy needed to overcome gravity was calculated with equation $\mathrm{W}=\mathrm{mgh}$ ( $\mathrm{m}$ is the mass of rover, $g$ is gravity and $h$ is height). Then total energy cost could be calculated. Suppose the weight of the rover is 25 kilograms, and the rolling resistance coefficient is 0.15 . In straight path planning the energy cost to overcome gravity is 406.7 joules. Otherwise in optimized path planning the energy cost to overcome gravity is 90.65 joules.
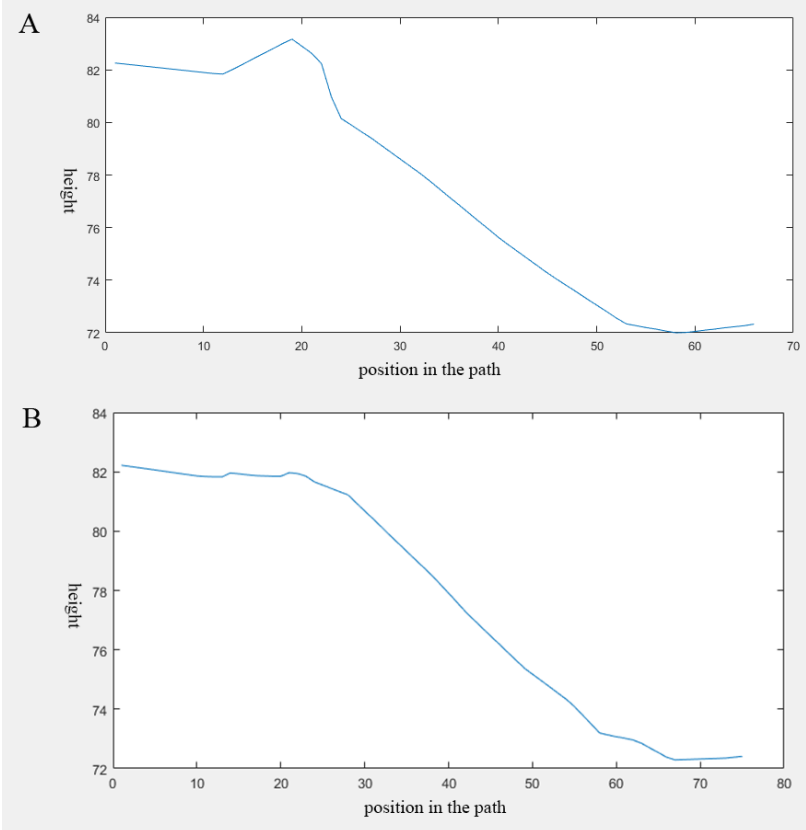

Figure 3. Diagram of height-position relationship for straight path (A) and energy optimized path (B). 
We assume the gravity and rolling resistance reach a balance in the downhill stage, so the energy cost to overcome rolling resistance only exist on the flat terrain or uphill. As shown in the straight-line path (Figure 3A), from waypoint 11 to 19 and 58 to 65 is uphill. And in the optimized path, the way from point 11 to 12,20 to 22 and 67 to 75 is uphill which is shorter than that of the straight path. So we assumed that at least $77 \%$ of the energy is saved.

Two simulations were performed to calculate the total energy needed. Under previous assumptions, no additional energy is needed in the downhill stage. The energy cost needed to overcome gravity and rolling resistance were calculated for the two paths using straight path planning and optimized path planning separately on the flat and uphill.

In uphill stage, the energy cost using straight path planning is 3224.2 joules. And using optimized path planning the energy cost is only 2494.1 joules. When moving on flat, 1240.4 joules and 1517.7 joules are needed for the straight-line path and optimized path respectively. So the total energy is 4464.6 joules using straight-line path planning and 4011.8 joules using optimized path planning. $10.15 \%$ of energy could be saved with our energy optimized path planning.

\section{Conclusion}

As shown in the simulations, most of the uphill terrain could be avoided and energy could be saved effectively with our optimized path planning. In total, more than $12 \%$ of energy was saved in the uphill stage, which is a dramatic decline.

In conclusion, the energy optimization path planning algorithm is a feasible solution to extend the battery life for field operated agricultural rover. What's more, this algorithm could be used to any battery-operated rover such as exoplanet exploration where the energy is even more precious.

\section{References}

1. C.C. Ooi, C. Schindelhauer, Mobile Networks \& Applications 14, 309-321 (2009).

2. S. Liu, D. Sun, IEEE/ASME Trans. Mechatronics 19, 401-411 (2014).

3. Y.K. Hwang, N. Ahuja, IEEE Trans. Robot. Autom 8, 23-32 (1992).

4. M.G. Park, J.H. Jeon, M.C. Lee, IEEE International Symposium on Industrial Electronics, 2001. Proceedings. ISIE 3, 1530-1535 (2001).

5. S.H. Suh, K.G. Shin, Robotics \& Automation IEEE Journal of 4, 334-349 (1988). 